\title{
Monolayer Properties of 1,3-Diamidophospholipids
}

\author{
lllya A. Fedotenko, ${ }^{\dagger}$ Cristina Stefaniu, ${ }^{\ddagger}$ Gerald Brezesinski, ${ }^{*} \neq$ and Andreas Zumbuehl ${ }^{*}, \S$ \\ ${ }^{\dagger}$ Department of Organic Chemistry, University of Geneva, Quai Ernest-Ansermet 30, 1211 Geneva, Switzerland \\ ${ }^{\star}$ Max Planck Institute of Colloids and Interfaces, Research Campus, Potsdam-Golm, 14476 Potsdam, Germany \\ ${ }^{\S}$ Department of Chemistry, University of Fribourg, Chemin du Musé 9, 1700 Fribourg, Switzerland
}

\begin{abstract}
While nature provides an endless variety of phospholipids presenting hydrolyzable ester linkages for the 1,2-positioned hydrocarbon tails, we designed and synthesized 1,3-diamidophospholipids which contain stable fatty acid amides. These new phospholipids form faceted unilamellar vesicles with mechanosensitive properties. Aiming to understand the mechanism responsible for this behavior at a molecular level, we investigated the 1,3-diamidophospholipid family in monolayers, a simplified model membrane system. Langmuir isotherms combined with in situ grazing incidence X-ray diffraction (GIXD), specular $\mathrm{X}$-ray reflectivity (XR), and infrared reflection-absorption spectroscopy (IRRAS) allowed the characterization of the monolayers from a structural and thermodynamical point of view. The existence of strong headgroup interactions due to the formation of a hydrogen-bonding network was clearly revealed by IRRAS and by the high rigidity of the monolayers. GIXD showed that only the longer chain compounds of the series (Pad-PC-Pad $(1,3-$ dipalmitamidopropan-2-phosphocholine) and Sad-PC-Sad (1,3-distearamidopropan-2-phos-

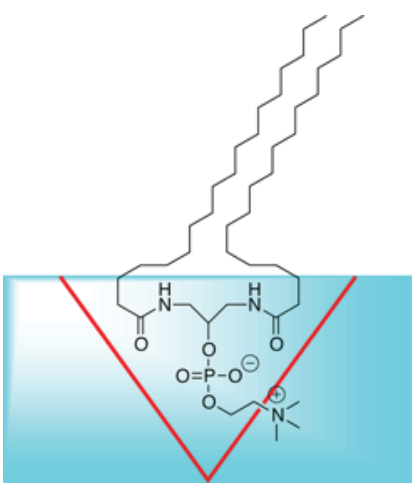
phocholine) were able to form ordered monolayers. The chains are strongly tilted in a rigid lattice formed due to these hydrogenbonding interactions between the headgroups. The thermodynamical analysis leads to a critical temperature of the monolayer which is clearly different from the main phase transition temperature in bulk, indicating that there must be a different structural arrangement of the 1,3-diamidophospholipids in monolayers and in bilayers.
\end{abstract}

\section{INTRODUCTION}

Nature provides an endless variety of phospholipids, and we are only just now starting to understand why this number is at all needed. ${ }^{1}$ The field of lipidomics has been fundamentally dusted off in recent years and helped science to reappreciate lipidology. ${ }^{2}$ One fact that is very appealing for an organic chemist is the need for constant adaptation to an ever-changing environment (homeoviscous adaptation). Therefore, nature focused on lipids made from easily interchangeable parts, which can be cleaved by enzymes and are based on ester bonds. ${ }^{3}$ This means, however, that there must be a vast chemical space that has not yet been (or maybe not anymore) touched upon by nature: These are phospholipids that contain stable amido fatty acid chains instead of ester hydrocarbon tails. ${ }^{4}$ Molecules that belong to this type of phospholipids are expected to selfassemble into membranes similar to natural lipids. Nonetheless, the modified chemical structures should lead to noticeable changes in membrane biophysics. Here, we present data highlighting features of artificial 1,3-diamidophospholipids confined in two dimensions (Langmuir monolayers).

Recently, we have noted the effect of the symmetric, artificial 1,3-diamidophospholipid Pad-PC-Pad (3) on the morphology of large unilamellar vesicles of $100 \mathrm{~nm}$ diameter prepared by the extrusion technique (LUVET ${ }_{100}$ ) and GUVs of $10 \mu \mathrm{m}$ diameter prepared by electroformation. ${ }^{4}$ Even though both vesicle types are significantly larger than $40 \mathrm{~nm}$, faceted vesicles were found below the main phase transition. We hypothesized that the interplay of the amido groups and the 1,3-substitution pattern would lead to a special vesicle organization with a continuous intermolecular hydrogen-bonding pattern. Therefore, we expanded our studies to a small library of the Xad-PCXad genus of phospholipids with tails ranging from $\mathrm{C} 12$ to $\mathrm{C} 18$ (see Figure 1, for nomenclature, see ref 5).

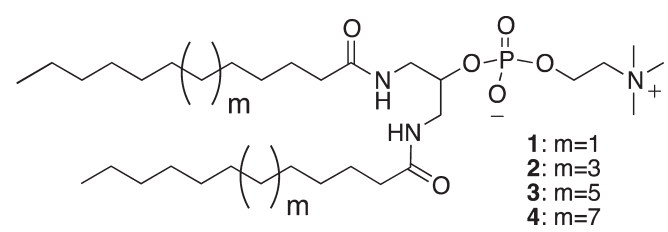

Figure 1. Xad-PC-Xad genus of 1,3-diamidophospholipids: Lad-PCLad (1), Mad-PC-Mad (2), Pad-PC-Pad (3), and Sad-PC-Sad (4).

It is interesting to note that the 1,3-diamidophospholipids form mechanosensitive vesicles: Vesicles that do not release their cargo spontaneously but do so when shaken. ${ }^{4}$ Such mechanosensitive vesicles represent an attractive tool for purely physics-based drug delivery systems using the human body's alteration of forces as a trigger for drug release, e.g., for targeting stenosed segments of the bloodstream. In order to 
understand the molecular basis of phenomena behind mechanosensitive drug delivery, the biophysical properties of 1,3-diamidophospholipid monolayers have been studied by surface sensitive techniques as grazing incidence $\mathrm{X}$-ray diffraction (GIXD), specular X-ray reflectivity (XR), and infrared reflection-absorption spectroscopy (IRRAS) to elucidate the structures formed in such 2D model systems confined to the air/water interface.

\section{RESULTS AND DISCUSSION}

Synthesis. The 1,3-diamidophosphocholines (1-4) were prepared from the corresponding cephalines. ${ }^{5}$ An exhaustive methylation of the phosphatidylethanolamine headgroup with dimethyl sulfate led to the cholines (see Supporting Information, Pad-PC-Pad (3) has already been reported in ref 17). The purification of the final compounds proved to be very difficult and could finally only be accomplished using a Sephadex LH-20 column. ${ }^{6}$

Differential scanning calorimetry of LUVET $_{100}$ revealed that the main transition temperature is increasing linearly with the length of the hydrocarbon chain $\mathrm{C}_{12}<273 \mathrm{~K} ; \mathrm{C}_{14} \approx 294 \mathrm{~K} ; \mathrm{C}_{16}$ $\approx 308 \mathrm{~K} ; \mathrm{C}_{18} \approx 325 \mathrm{~K}$.

Surface Pressure/Molecular Area Isotherms. Pressurearea isotherms of monolayers of the compounds $\mathbf{1 - 4}$ have been measured at the air-water interface at $293 \mathrm{~K}$ (Figure 2). As

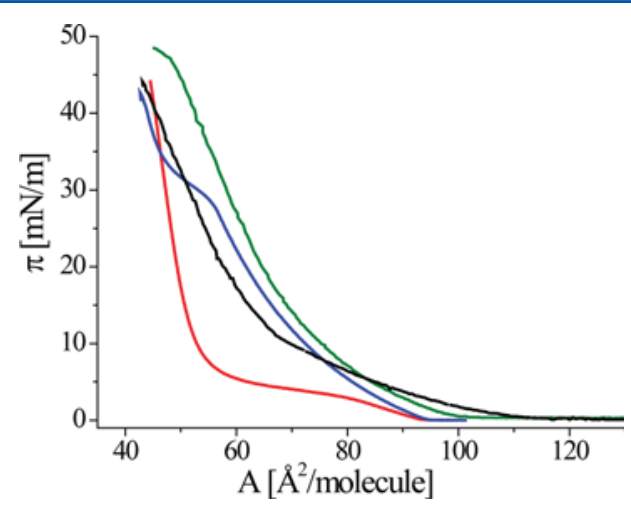

Figure 2. $\pi-A$ isotherms of Lad-PC-Lad (1, black line) and Mad-PCMad (2, green line), Pad-PC-Pad (3, blue line), and Sad-PC-Sad (4, red line) monolayers on water measured at $293 \mathrm{~K}$.

expected from the low main phase transition temperature $\left(T_{\mathrm{m}}\right)$ values, both Lad-PC-Lad (1) and Mad-PC-Mad (2) form only liquid-expanded monolayers with typical molecular areas around $60 \AA^{2}$ at $30 \mathrm{mN} \mathrm{m}^{-1}$. Compression up to high lateral pressures does not lead to the formation of an ordered condensed phase in the accessible temperature range. Compound $\mathbf{1}$ has apparently a smaller molecular area at higher lateral pressures compared to compound 2 . This shows that the monolayer stability of the shorter-chain compound is lower with a partial solubility at higher compression of the film. On the contrary, the isotherms of Pad-PC-Pad (3) and Sad-PC-Sad (4) show a plateau region characterizing the first-order phase transition from the fluid (liquid-expanded (LE)) to the condensed (liquid-condensed (LC)) phase. As observed for many amphiphilic monolayers, the theoretically horizontal twophase transition region is increasingly inclined at higher transition pressures as seen for Pad-PC-Pad (3). The transition pressure $\pi_{\mathrm{t}}$ amounts to $28.8 \mathrm{mN} \mathrm{m}^{-1}$ for compound 3 and to $2.8 \mathrm{mN} \mathrm{m}^{-1}$ for compound 4. As expected, the transition pressure increases with decreasing chain length or equivalent with increasing temperature. All films are rather stable up to lateral pressures above $40 \mathrm{mN} \mathrm{m}^{-1}$.

Based on these results, pressure-area isotherms have been measured at various temperatures. Even at $278 \mathrm{~K}$, both Lad-PCLad (1) and Mad-PC-Mad (2) form only liquid-expanded monolayers. Compression does not lead to the formation of an ordered condensed phase. GIXD experiments do not show Bragg peaks of an ordered structure, and IRRAS demonstrates the fluid conformation of the chains by high wavenumbers of the $\mathrm{CH}_{2}$ stretching bands. On the contrary, the isotherms of Pad-PC-Pad (3) and Sad-PC-Sad (4) show at most studied temperatures the plateau region characterizing the coexistence of two phases (Figure 3). The temperature dependence of the transition pressure $\pi_{\mathrm{t}}$ can be described by a linear function (Figure 4), from which the experimental data points deviate only in the vicinity of $T_{0}$ which determines the lowest temperature of the existence of the liquid-expanded phase. Below this temperature, the transition into the condensed phase starts directly from the gas-analogous state. $T_{0}$ amounts to $291 \mathrm{~K}$ for Sad-PC-Sad and to $274.6 \mathrm{~K}$ for Pad-PC-Pad. The slope $\mathrm{d} \pi_{t} / \mathrm{d} T$ of the linear function is $1.544 \mathrm{mN}(\mathrm{m} \mathrm{K})^{-1}$ for Pad-PC-Pad and $1.322 \mathrm{mN}(\mathrm{m} \mathrm{K})^{-1}$ for Sad-PC-Sad.

The two-dimensional Clausius-Clapeyron equation representing a one-component approximation can be used for calculating the enthalpy change $\Delta H$ of the phase transition

$$
\Delta H=\left(A_{\mathrm{c}}-A_{\mathrm{e}}\right) T \frac{\mathrm{d} \pi_{t}}{\mathrm{~d} T}
$$
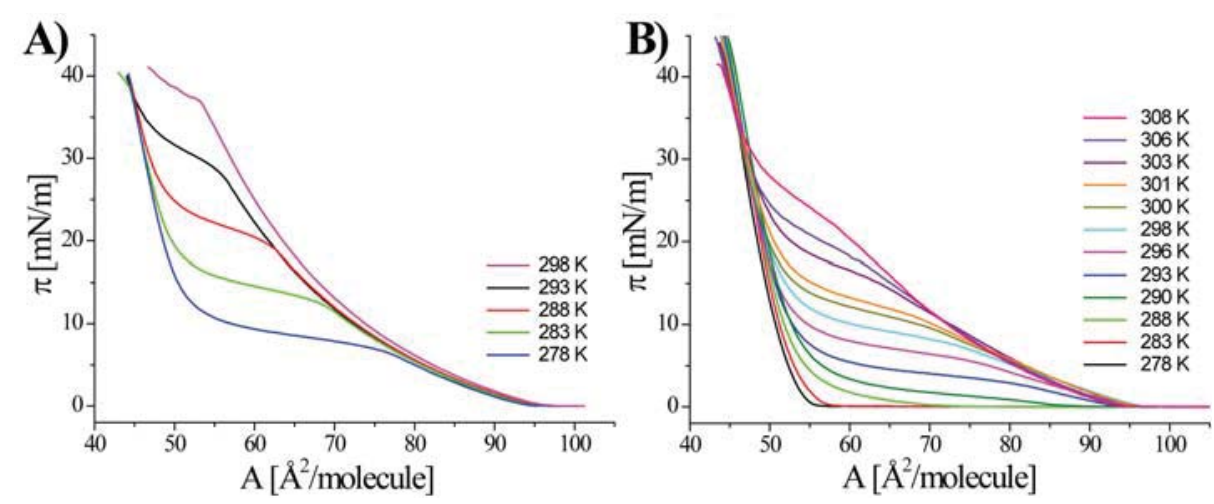

Figure 3. $\pi-A$ isotherms of Pad-PC-Pad (A) and Sad-PC-Sad (B) monolayers on water measured in the temperature range between 278 and $308 \mathrm{~K}$. 

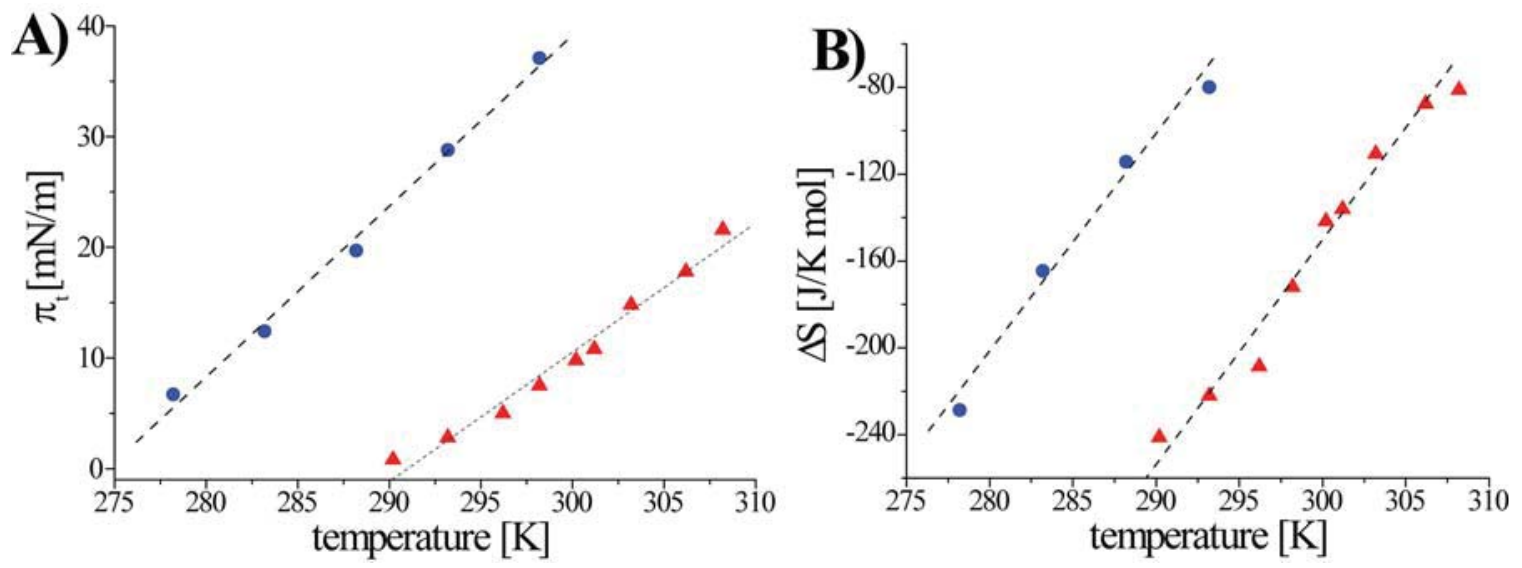

Figure 4. (A) Temperature dependence of the main phase transition pressure $\pi_{t}$ of Pad-PC-Pad (blue $\mathbf{0}$ ) and Sad-PC-Sad (red $\mathbf{\Delta}$ ) monolayers spread on water. (B) Temperature dependence of the entropy change at the main phase transition of Pad-PC-Pad (blue $\mathbf{0}$ ) and Sad-PC-Sad (red $\mathbf{\Delta}$ ) monolayers spread on water.
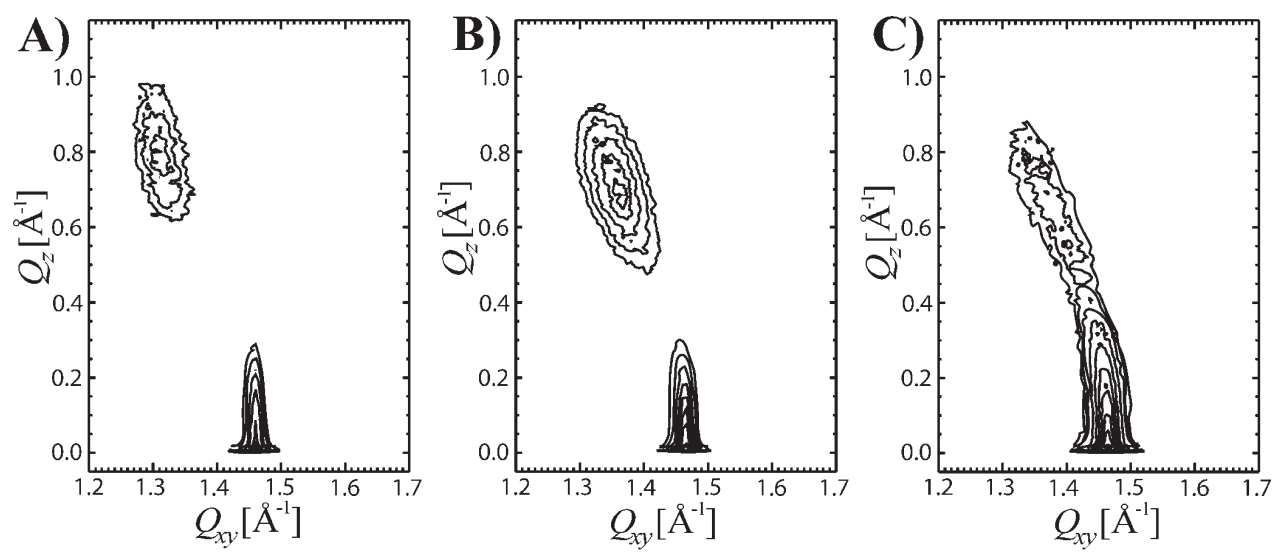

Figure 5. Grazing incidence X-ray diffraction data for Sad-PC-Sad spread on water at $293 \mathrm{~K}$ and compressed to 10 (A), 20 (B), and 35 (C) $\mathrm{mN} \mathrm{m}{ }^{-1}$. The diffracted intensity, corrected for polarization, effective area, and Lorentz factor, is plotted as contour lines of equal intensity versus the in-plane component $Q_{x y}$ and the out-of-plane component $Q_{z}$ of the scattering vector.

where $A_{\mathrm{e}}$ is the molecular area at the onset of the phase transition at the surface pressure $\pi_{t}$ and $A_{c}$ is the area of the condensed phase at this pressure. ${ }^{7}$

The temperature dependence of the entropy change $\Delta S=$ $\Delta H / T$ for the phase transition is presented in Figure 4B. Negative $\Delta H$ and $\Delta S$ values are obtained according to the exothermic nature of the main phase transition at compression of amphiphilic monolayers and an increase in the ordering of the system. The absolute $\Delta S$ and $\Delta H$ values increase as the temperature decreases, indicating that the ordering of the condensed phase increases as the temperature decreases. The critical temperature of $300.4 \mathrm{~K}$ for Pad-PC-Pad and 314.5 K for Sad-PC-Sad, above which the monolayer cannot be compressed to the condensed state, results from the extrapolation of $\Delta S$ to zero. The values are considerably lower than the main transition temperatures in bulk (308 and $325 \mathrm{~K}$, respectively), and the difference increases with increasing chain length, indicating that the packing in $3 \mathrm{D}$ bilayers must be different compared to that in 2D monolayers.

Grazing Incidence X-ray Diffraction (GIXD) and Specular X-ray Reflectivity (XR). GIXD studies provide information about lattice structures of condensed monolayer phases on the angstrom scale. ${ }^{8,9}$ Figure 5 shows selected contour plots of equal intensity versus the in-plane component
$Q_{x y}$ and the out-of-plane component $Q_{z}$ of the scattering vector at $293 \mathrm{~K}$

In the case of Sad-PC-Sad, three Bragg peaks (the two ones at higher $Q_{z}$ values are strongly overlapping and the third one is slightly above the horizon) have been observed at all pressures at $293 \mathrm{~K}$, showing that the structure of the condensed monolayer phase is oblique with strongly tilted chains (Figure $5)$. Because the two Bragg peaks at higher $Q_{z}$ values are strongly overlapping, the structure is close to an orthorhombic one with NN (nearest neighbor) tilted chains. The chain crosssectional area is between 19.9 and $20.0 \AA^{2}$ often found for longchain phospholipids as PCs. These are typical values for a rotator phase indicating free rotation of the alkyl chains. The tilt angle $t$ with respect to the surface normal decreases only marginally with increasing pressure: $0.26^{\circ}$ per $\mathrm{mN} \mathrm{m}^{-1}$ (see Supporting Information, Figure S1). The transition pressure into a nontilted phase can be calculated using the following consideration: Since the condensed isotherms exhibit linear relations between pressure and molecular area and assuming a constant cross-sectional area in the condensed phase, plotting of $1 / \cos (t)$ as a function of the lateral pressure allows the determination of the tilting phase transition pressure by extrapolation toward $1 / \cos (t)=1 .^{9}$ At $293 \mathrm{~K}$, the nontilted phase can be expected only at unattainable high pressure values $\left(\sim 148 \mathrm{mN} \mathrm{m}^{-1}\right)$. Decreasing the temperature does not change 

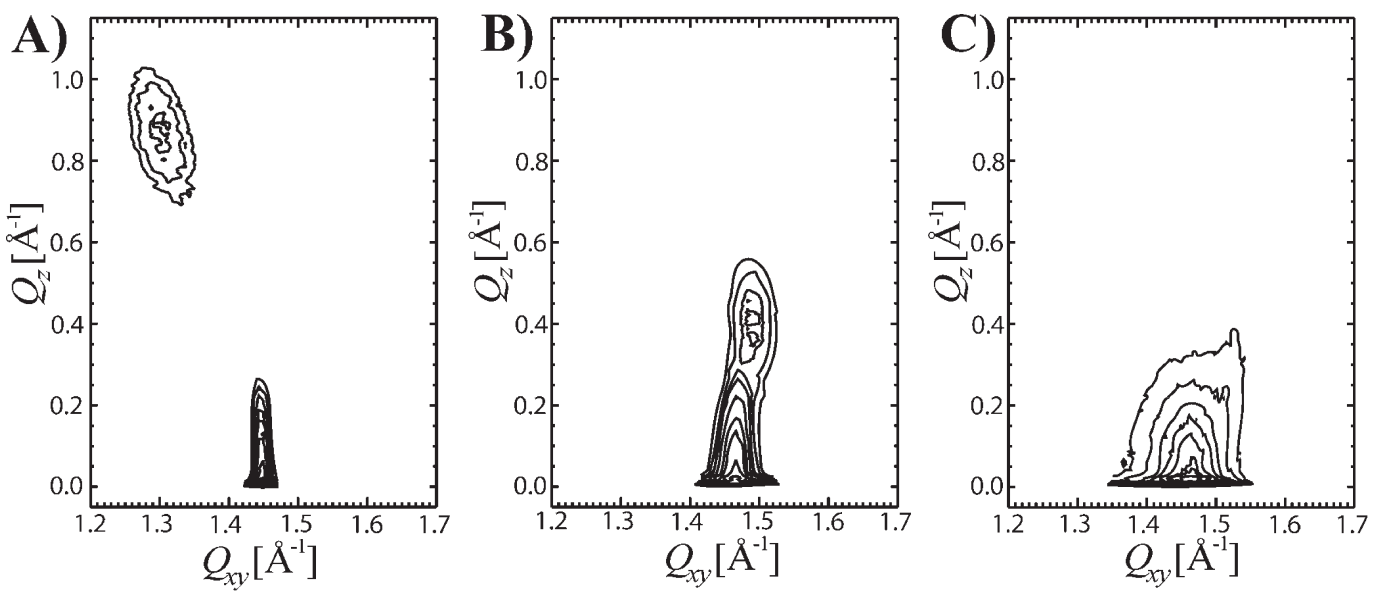

Figure 6. Grazing incidence X-ray diffraction data for Sad-PC-Sad spread on water at $278 \mathrm{~K}$ and compressed to 10 (A) and 35 (B) $\mathrm{mN} \mathrm{m}^{-1}$. The diffracted intensity, corrected for polarization, effective area, and Lorentz factor, is plotted as contour lines of equal intensity versus the in-plane component $Q_{x y}$ and the out-of-plane component $Q_{z}$ of the scattering vector. The diffraction pattern is drastically changed when the sample is kept in the compressed state at $35 \mathrm{mN} \mathrm{m}^{-1}$ for $2 \mathrm{~h}(\mathrm{C})$.

the packing properties. Also at $278 \mathrm{~K}$, three Bragg peaks characteristic of an oblique condensed monolayer have been observed. As expected, the cross-sectional area is decreased $\left(19.5 \AA^{2}\right)$. At high compression, the lattice changes to an orthorhombic one with chains tilted in the $\mathrm{NN}$ (nearest neighbor) direction and a NNN (next-nearest neighbor) distortion (Figure 6). Surprisingly, the tilt angle decreases on compression in a more pronounced way. The value of $0.68^{\circ}$ per $\mathrm{mN} \mathrm{m}{ }^{-1}$ is almost 3 times larger than the one observed at 293 $\mathrm{K}$. Therefore, at $278 \mathrm{~K}$, the tilting phase transition pressure amounts to $\sim 43 \mathrm{mN} \mathrm{m}^{-1}$, showing that the nontilted phase can be expected at attainable pressure values. In most cases, such a tilting transition between two condensed phases is a secondorder phase transition, usually characterized by a kink in the isotherm. However, the expected kink is not clearly visible in the isotherm (see Figure 3). This could be a general problem of measuring the surface tension by the Wilhelmy plate method in the case of stiff monolayers causing the tilting of the plate.

Additionally, the diffraction pattern changes drastically if the monolayer is kept in the compressed state at $35 \mathrm{mN} \mathrm{m}^{-1}$ for 2 h. The monolayer structure is still orthorhombic, but the tilt angle is now much lower $\left(9^{\circ}\right.$ instead of $\left.18^{\circ}\right)$. This tilt angle decrease is connected with an increase in the cross-sectional area. One possibility could be the breaking of intermolecular hydrogen bonds between the headgroups. This gives more rotational freedom to the chains what leads to a less tilted state with still optimized van der Waals interactions between the chains. We will discuss the formation of hydrogen bonds in the IRRAS section, but unfortunately intra- and intermolecular hydrogen bonds as well as the ones formed between the headgroups or between the headgroups and water molecules cannot be distinguished.

The electron density profile perpendicular to the water surface has been determined by specular X-ray reflectivity (XR) experiments (Supporting Information). ${ }^{10}$ The model-free inversion of the reflectivity data yields the laterally averaged electron density $\rho(z)$ of the monolayer as a function of the vertical $z$ coordinate. Using the molecular area of Sad-PC-Sad and the known number of electrons in the hydrophobic (274 $\left.\mathrm{e}^{-}\right)$and hydrophilic $\left(157 \mathrm{e}^{-}\right)$parts of the molecule, the electron density profile has been described by two Gaussian shaped curves. The fwhm of the curve representing the hydrophobic chain region amounts to $19.2 \AA$, which translates directly into the thickness of this layer. The second layer has a thickness of $12.1 \AA$ with a maximum electron density of $0.406 \mathrm{e}^{-} / \AA^{3}$. The expected length of a fully stretched $\mathrm{C} 18$ chain in all-trans conformation amounts to $21.6 \AA$. The difference between these two values leads to a theoretical tilt angle of $27^{\circ}$ (assuming that the whole chain is in all-trans conformation) which is larger than the one determined by GIXD. This shows that the chains are not parallel to each other over the whole chain length in 1,3-diamidophospholipids due to geometrical packing restrictions. On the basis of the tilt angle of $18^{\circ}$, as determined by GIXD, a thickness of $20.5 \AA$ could be expected, showing that a certain part of the chain region must be disordered and contributes therefore with less than $1.26 \AA$ per $\mathrm{C}-\mathrm{C}$ (characteristic for an all-trans conformation) ${ }^{11,12}$ to the chain length determined by XR experiments. In contrast to the GIXD experiments, which characterize only well-ordered monolayer structures, $\mathrm{XR}$ is sensitive to ordered as well as to unordered monolayer parts. The model of ordering only a part of the chain is also supported by the fwhm $\left(0.32 \AA^{-1}\right)$ of the Bragg rod. This value can be translated into the length of the scatterer and amounts to $17.7 \AA$ corresponding to a fully stretched C14 chain, showing again that only the upper part of the chain is forming the observed chain lattice. Because of the 1,3substitution pattern, the first methylene groups close to the headgroup region might be by sterical reasons hindered to be close enough and perfectly parallel to each other to optimize their van der Waals interactions (cone shape). These groups do not contribute to the diffraction pattern (GIXD) of the chain lattice and give a smaller contribution to the layer thickness (XR) than a fully stretched C18 chain. This might be also the reason for the quite high wavenumbers of the $\mathrm{CH}_{2}$-stretching vibrations (see IRRAS part), showing that some of these groups are not in a tightly packed all-trans conformation.

The contour plot of Pad-PC-Pad shows that the diffracted intensity is distributed along an arc (Figure 7). On moving to larger $Q_{z}$ values, the maximum position is shifted to lower $Q_{x y}$ values while the peak width increases. The intensity curve does not follow the so-called Scherrer lines, which are lines of identical $Q$-values. This is good proof that the diffraction pattern is not caused by three-dimensional crystals from collapsed material, but by regular monomolecular layers. The 


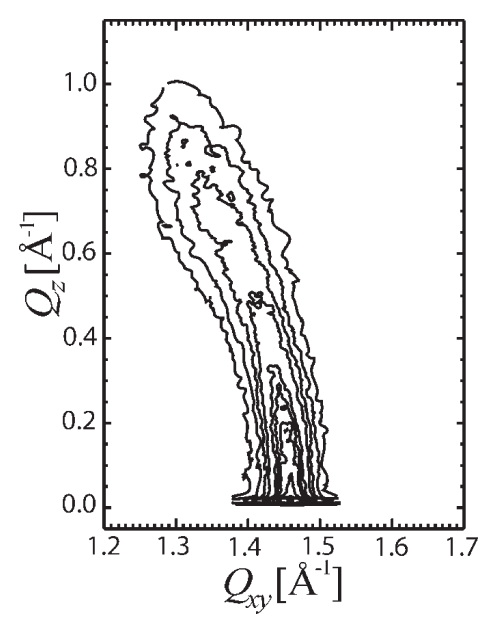

Figure 7. Grazing incidence X-ray diffraction data for Pad-PC-Pad (3) spread on water at $278 \mathrm{~K}$ and compressed to $35 \mathrm{mN} \mathrm{m}^{-1}$. The diffracted intensity, corrected for polarization, effective area, and Lorentz factor, is plotted as contour lines of equal intensity versus the in-plane component $Q_{x y}$ and the out-of-plane component $Q_{z}$ of the scattering vector.

observed diffraction pattern is assigned to be a superposition of reflections from several lattices within the monolayers. In an earlier study, we have shown that such a distributed scattered intensity can be unambiguously ascribed to a variation of the tilt azimuth. ${ }^{13}$ A possible variation of the tilt angle could be excluded. The tilt azimuth is defined as the angle between the projection of the tilted chain on the surface plane and the lattice axis, whereas the tilt angle is the angle between the aliphatic chain and the surface normal. The peak at zero $Q_{z}$ should be the (02) reflection of an orthorhombic phase with chains tilted toward nearest neighbors $(\mathrm{NN})$, and that at the largest $Q_{z}$ should be the (02) reflection of a next-nearest-neighbor (NNN) tilted structure. The corresponding degenerate peak of the NN tilted lattice, located at larger $Q_{z}$ values, cannot be so easily determined. However, this is possible for the degenerate Bragg peak of the NNN lattice. The degenerate peak should have its intensity maximum at half the $Q_{z}$ value of the nondegenerate peak. The accuracy of the determination of peak positions is not as high as in the case of Sad-PC-Sad. Between the limits of the two orthorhombic phases ( $\mathrm{NN}$ and $\mathrm{NNN}$ ), several oblique phases with the same tilt angle but different tilt azimuths contribute to the observed scattering profile. The tilt angle determined from the data of the NNN lattice amounts to $31.5^{\circ}$. The cross-sectional area is $20.2 \AA^{2}$ and therefore slightly larger compared to Sad-PC-Sad with the longer chains. Very similar values have been observed for DPPC and DSPC, showing that the packing density of the 1,2-glycerophospholipids is similar to that of 1,3-diamidophospholipids. Obviously, the large and strongly hydrated PC headgroup plays the major role by defining the area requirement of the molecules. The reason for the undefined tilt direction of the shorter-chain compound Pad-PC-Pad compared to the longer-chain compound Sad-PC-Sad could be the molecular shape. Since the two chains are connected with one $\mathrm{C}$ atom, the molecule could exhibit a cone shape close to the headgroup region (the 1,3substitution pattern leads to a large headgroup-to-tail diameter ratio) with effectively shorter chains aligned parallel to each other in the hydrophobic part of the molecules (see discussion above). On the other hand, the molecules are able to form hydrogen bonds to stabilize the monolayer structure. The longer chains with increased van der Waals interactions are better able to optimize the packing properties with a defined tilt direction in a lattice in which the dimensions are dictated by the headgroups.

Infrared Reflection-Absorption Spectroscopy. In order to get additional information about the molecular interactions, infrared reflection-absorption spectroscopy (IRRAS) experiments were performed on monolayers of PadPC-Pad (3) and Sad-PC-Sad (4). ${ }^{14-17}$ The spectra recorded revealed changes occurring in the organization of the hydrocarbon chains upon an increase of the molecular packing as well as information about the headgroup interactions and hydration state. Thus, the spectra obtained in the $\mathrm{CH}$ stretching region (Figure 8) show that upon increasing the lateral surface pressure, the band positions for both symmetric and asymmetric methylene stretching vibrations shift to lower wavenumbers. This is in perfect accordance with the occurrence of the two-phase plateau region in the corresponding isotherms (Figure 3). The phase transition of the alkyl chains leads from a liquid (LE) disordered state (gauche conformation of the chains) to an ordered LC state (all-trans conformation of the alkyl chains). ${ }^{17,18}$ However, as discussed above, lower wavenumbers have been expected in such a tightly packed layer
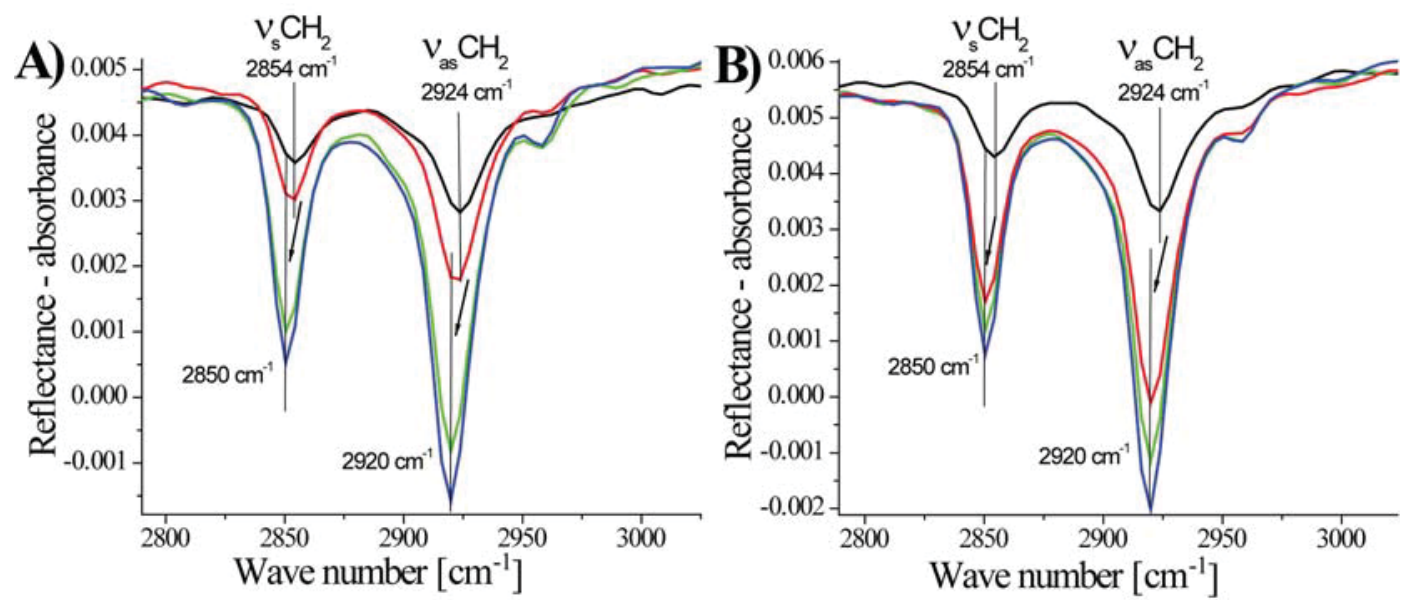

Figure 8. CH stretching region of IRRA spectra of Pad-PC-Pad at $280 \mathrm{~K}(\mathrm{~A})$ and of Sad-PC-Sad at $293 \mathrm{~K}(\mathrm{~B})$ at different surface pressures $(1 \mathrm{mN}$ $\mathrm{m}^{-1}$, black; $10 \mathrm{mN} \mathrm{m}^{-1}$, red; $20 \mathrm{mN} \mathrm{m}^{-1}$, green; $35 \mathrm{mN} \mathrm{m}^{-1}$, blue) of monolayers prepared on water (p-polarized light, incidence angle $40^{\circ}$ ). 

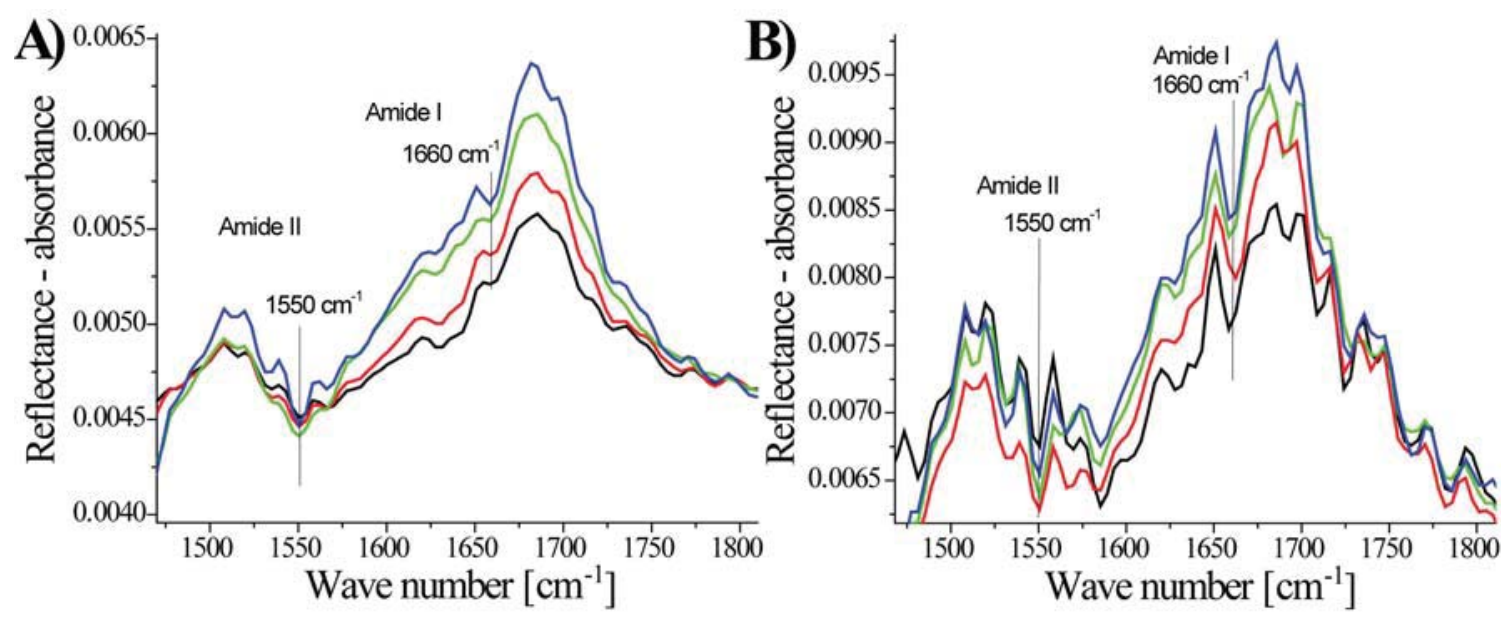

Figure 9. Amide I and amide II regions of IRRA spectra of Pad-PC-Pad at $280 \mathrm{~K}$ (A: s-polarized light, incidence angle $40^{\circ}$ ) and of Sad-PC-Sad at $293 \mathrm{~K}$ (B: p-polarized light, incidence angle $\left.40^{\circ}\right)$ at different surface pressures $\left(1 \mathrm{mN} \mathrm{m}^{-1}\right.$, black; $10 \mathrm{mN} \mathrm{m}^{-1}$, red; $20 \mathrm{mN} \mathrm{m}-1$, green; $35 \mathrm{mN} \mathrm{m}{ }^{-1}$, blue) of monolayers prepared on water.
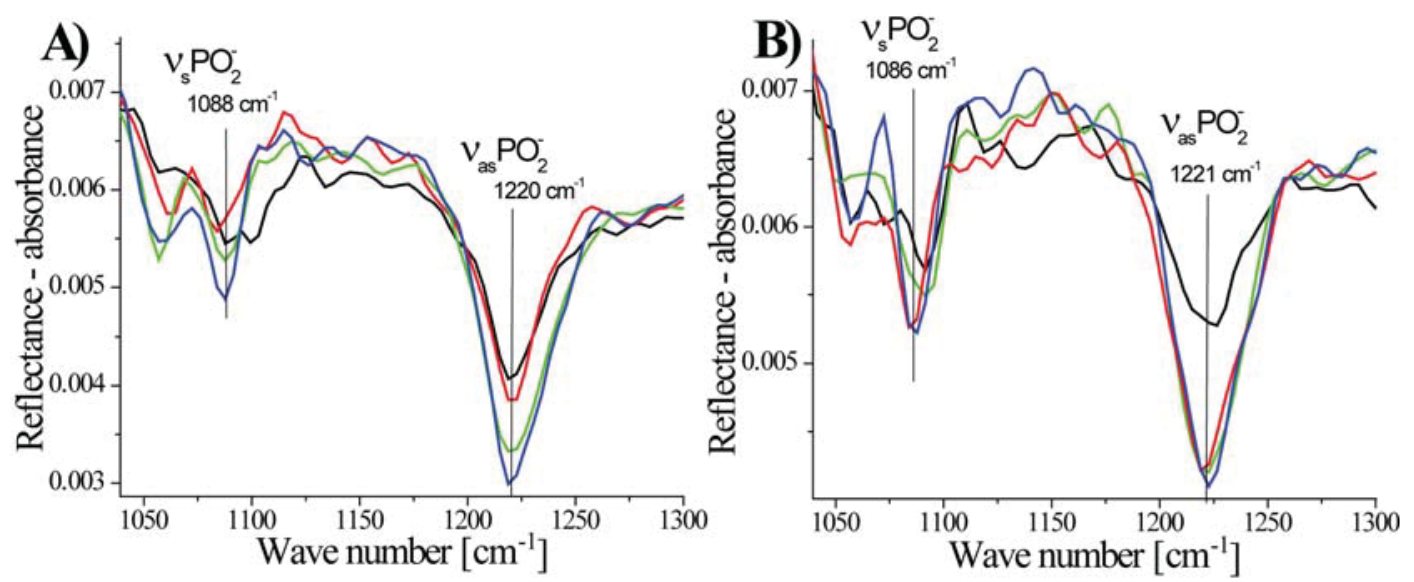

Figure 10. Phosphate stretching region of region of IRRA spectra of Pad-PC-Pad at $280 \mathrm{~K}$ (A) and of Sad-PC-Sad at $293 \mathrm{~K}$ (B) at different surface pressures $\left(1 \mathrm{mN} \mathrm{m}^{-1}\right.$, black; $10 \mathrm{~m} \mathrm{~m}^{-1}$, red; $20 \mathrm{mN} \mathrm{m}^{-1}$, green; $35 \mathrm{mN} \mathrm{m}^{-1}$, blue) of monolayers prepared on water (p-polarized light, incidence angle $40^{\circ}$ ).

stabilized by a H-bond network. The reason might be the contribution of some $\mathrm{CH}_{2}$ groups between the hydrophilic headgroup and the well-packed hydrophobic chains which are not as close to the neighboring chains as needed for a perfect all-trans conformation due to the geometrical restrictions of the 1,3-substitution pattern.

The high rigidity of the monolayers, expressed by the marginal change of the strong tilting of the alkyl chains on compression (Supporting Information), could be explained by the existence of strong headgroup interactions. The amide groups are the moieties forming hydrogen bonds with the neighboring lipid molecules or with water. It is well-known that such $\mathrm{H}$-bonds shift the amide I band (mostly carbonyl vibration $\nu(\mathrm{C}=\mathrm{O}))$ to lower wavenumbers and the amide II band (primarily $\mathrm{NH}$ bending vibration) in the opposite direction. Since the band position is very sensitive to molecular association, the amide I band appears in inert solutions $\left(\mathrm{CCl}_{4}\right)$ (no $\mathrm{NH} \cdots \mathrm{O}=\mathrm{C}$ hydrogen bonds) at $1700-1670 \mathrm{~cm}^{-1}$. This band shifts in the solid state to $1680-1630 \mathrm{~cm}^{-1}$. For the studied monolayers the amide I band was recorded for both compounds at $1660 \mathrm{~cm}^{-1}$, as a very weak band, especially for Pad-PC-Pad.
The amide II band that is specific for the noncyclic secondary amides appears in the range $1540-1510 \mathrm{~cm}^{-1}$ for dilute inert solutions and at $\sim 1550 \mathrm{~cm}^{-1}$ for the solid state. For the monolayers of both Pad-PC-Pad and Sad-PC-Sad, the IRRA spectra (Figure 9) revealed a band centered at $1550 \mathrm{~cm}^{-1}$, namely, at the position characteristic for hydrogen bonding. ${ }^{19-22}$

Thus, the IRRA spectra show that the amide moieties are hydrogen bonded in the monolayers of both compounds. These findings correlated with the rigidity of the layer indicate that the headgroups are interconnected by hydrogen bonds. Despite the H-bond network, no order of the headgroups could be observed for these compounds, as recently reported for monolayers of a GPI fragment. ${ }^{23}$

Concerning the headgroup region, the IRRA spectra additionally revealed the symmetric and asymmetric $\mathrm{PO}_{2}^{-}$ double-bond stretching bands occurring at $1086(1088) \mathrm{cm}^{-1}$ and $1221 \mathrm{~cm}^{-1}$, respectively (Figure 10). The frequency of $1221 \mathrm{~cm}^{-1}$ is considered to characterize a fully hydrated phosphate group. ${ }^{24}$ No shift of this band position was observed upon lateral compression of the monolayer, indicating a lack of change in the headgroup hydration state. This behavior is probably due to the formation of the rigid $\mathrm{H}$-bond network and 
is contrary to the previously observed dehydration of phospholipid headgroups upon monolayer compression. ${ }^{25,26}$

\section{CONCLUSIONS}

This study was conducted with the aim of identifying at a molecular scale the structures and specific interactions in monolayers of newly synthesized 1,3-diamidophospholipids. The next step will be the comparison of $2 \mathrm{D}$ (monolayers) and 3D (bilayers) structures to understand the ability of forming faceted unilamellar vesicles. The monolayers were characterized from a thermodynamical and structural point of view. With respect to their degree of organization, the new 1,3diamidophospholipids showed a classical behavior. Namely, the monolayers formed by the shorter-chain compounds (LadPC-Lad (1) and Mad-PC-Mad (2)) are only in a liquidexpanded (LE) state, while the longer-chain compounds (PadPC-Pad (3) and Sad-PC-Sad (4)) are able to form condensed monolayers. These findings are in agreement with the fact that only Pad-PC-Pad and Sad-PC-Sad, being in a gel state at room temperature, were able to form faceted vesicles. GIXD revealed a unique, homogeneous structure at different lateral pressures. The existence of strong headgroup interactions due to the formation of a hydrogen bond network was clearly revealed by IRRAS and by the high rigidity of the layers. This rigidity is correlated to the marginal change of the chain tilt upon compression of the monolayer. Such strong hydrogen-bonding interactions between the headgroups could be also responsible for the observed faceted shape in $3 \mathrm{D}$ aggregates. However, there is a clear difference between monolayers and bilayers of 1,3-diamidophospholipids as the critical temperature of the monolayer is not equal to but much lower than the main phase transition temperature of the bilayer. We suggest that this could be due to membrane interdigitation that would only be possible in the case of bilayers. ${ }^{27-32}$ This would lead to a high lateral membrane stability, which would suggest that vesicles formulated from 1,3-diamidophospholipids would be prone to show facets.

It is interesting to note that the lipid chains of 1,3diamidophospholipids are spread further apart than the chains of natural 1,2-phospholipids. This together with the fact that the mean molecular area of the lipids is governed by strong hydrogen-bonding interactions between the headgroups leads to an undefined tilt direction in monolayers of the shorter-chain compounds. Only above a certain chain length threshold, allowing stronger van der Waals interactions between the chains, lattices with a defined tilt and a defined tilt direction are observed.

\section{ASSOCIATED CONTENT}

\section{S Supporting Information}

Experimental details. This material is available

\section{AUTHOR INFORMATION}

\section{Corresponding Author}

*E-mail: brezesinski@mpikg.mpg.de (G.B.); andreas. zumbuehl@unifr.ch (A.Z.).

\section{Notes}

The authors declare no competing financial interest.

\section{ACKNOWLEDGMENTS}

This work was supported by the Swiss National Science Foundation (Grants PP00P2_138926/1 and 200020_132035 to A.Z.) and European Cooperation in Science and Technology Action CM1101. The authors thank the Bioimaging, MS, and NMR facilities at the University of Geneva for analytical services. We thank HASYLAB at DESY, Hamburg, Germany, for beam time and excellent support. This work was partially supported by a Max Planck Society fellowship granted to C.S.

\section{REFERENCES}

(1) Dowhan, W. Molecular basis for membrane phospholipid diversity: why are there so many lipids? Annu. Rev. Biochem. 1997, 66, 199-232.

(2) Mouritsen, O. G. Lipidology and lipidomics--quo vadis? A new era for the physical chemistry of lipids. Phys. Chem. Chem. Phys. 2011, 13, 19195-19205

(3) Sinensky, M. Homeoviscous adaptation-a homeostatic process that regulates the viscosity of membrane lipids in Escherichia coli. Proc. Natl. Acad. Sci. U. S. A. 1974, 71, 522-525.

(4) Holme, M. N.; Fedotenko, I. A.; Abegg, D.; Althaus, J.; Babel, L.; Favarger, F.; Reiter, R.; Tanasescu, R.; Zaffalon, P. L.; Ziegler, A.; Müller, B.; Saxer, T.; Zumbuehl, A. Shear-stress sensitive lenticular vesicles for targeted drug delivery. Nat. Nanotechnol. 2012, 7 (8), 53643.

(5) Fedotenko, I. A.; Zaffalon, P. L.; Favarger, F.; Zumbuehl, A. The synthesis of 1,3-diamidophospholipids. Tetrahedron Lett. 2010, 51 (41), 5382-5384.

(6) Da Silva, V., personal communication.

(7) Glazer, J.; Alexander, A. E. The monolayer polymorphism of hexadecyl and octadecyl urea. Trans. Faraday Soc. 1951, 47, 401-409.

(8) Kaganer, V. M.; Möhwald, H.; Dutta, P. Structure and phase transitions in Langmuir monolayers. Rev. Mod. Phys. 1999, 71, 779819.

(9) Kjaer, K. Some simple ideas on X-ray reflection and grazingincidence diffraction from thin surfactant films. Physica B 1994, 198, $100-109$.

(10) Helm, C. A.; Möhwald, H.; Kjaer, K.; Als-Nielsen, J. Phospholipid monolayer density distribution perpendicular to the water surface. A synchrotron X-ray reflectivity study. Europhys. Lett. 1987, 4, 697-708.

(11) Israelachvili, J. N. Intermolecular and Surface Forces, 3rd ed.; Academic Press: San Diego, CA, 2011.

(12) Kitaigorodskii, A. I. Organic Chemical Crystallography; Consultants Bureau: New York, 1961.

(13) Weidemann, G.; Brezesinski, G.; Vollhardt, D.; Möhwald, H. Disorder in Langmuir monolayers. 1. Disordered packing of alkyl chains. Annu. Rev. Phys. Chem. 1995, 46, 305-334.

(14) Dluhy, R.; Cornell, D. G. In situ measurement of the infrared spectra of insoluble monolayers at the air-water interface. J. Phys. Chem. 1985, 89, 3195-3197.

(15) Mendelsohn, R.; Brauner, J. W.; Gericke, A. External infrared reflection absorption spectrometry of monolayer films at the air-water interface. Annu. Rev. Phys. Chem. 1995, 46, 305-334.

(16) Flach, C. R.; Brauner, J. W.; Taylor, J. W.; Baldwin, R. C.; Mendelsohn, R. External reflection FTIR of peptide monolayer films in situ at the air/water interface: experimental design, spectra-structure correlations, and effects of hydrogen-deuterium exchange. Biophys. J. 1994, 67, 402-410.

(17) Mendelsohn, R.; Mao, G.; Flach, C. R. Infrared reflectionabsorption spectroscopy principles and applications to lipid-protein interaction in Langmuir films. Biochim. Biophys. Acta 2010, 1798, 788800.

(18) Buontempo, J. T.; Rice, S. A. Infrared external reflection spectroscopic studies of phase transitions in Langmuir monolayers of heneicosanol. J. Chem. Phys. 1993, 98, 5835-5846. 
(19) Tam-Chang, S.-W.; Biebuyck, H. A.; Whitesides, G. M.; Jeon, N.; Nuzzo, R. G. Self-assembled monolayers on gold generated from alkanethiols with the structure $\mathrm{RNHCOCH}_{2} \mathrm{SH}$. Langmuir 1996, 11, 4371-4382.

(20) Bellamy, L. J. The Infrared Spectra of Complex Molecules, 2nd ed.; John Wiley \& Sons: New York, 1958; pp 203-233.

(21) Lee Smith, A. In Applied Infrared Spectroscopy. Fundamentals, Techniques, and Analytical Problem Solving; Elving, P. J., Winefordner, J. D., Eds.; John Wiley \& Sons: New York, 1979; Vol. 54, p 290, Appendix 2.

(22) Andreeva, T. D.; Petrov, J. G.; Brezesinski, G.; Möhwald, H. Structure of the langmuir monolayers with fluorinated ethyl amide and ethyl ester polar heads creating dipole potentials of opposite sign. Langmuir 2008, 24, 8001-8007.

(23) Stefaniu, C.; Vilotijevic, I.; Santer, M.; Silva, D. V.; Brezesinski, G.; Seeberger, P. H. Subgel phase structure in monolayers of glycosylphosphatidylinositol glycolipids. Angew. Chem., Int. Ed. 2012, 51, 12874-12878.

(24) Dluhy, R. A.; Cameron, D. G.; Mantsch, H. H.; Mendelsohn, R. Fourier transform infrared spctroscopic studies of the effect of calcium ions on phosphatidylserine. Biochemistry 1983, 22, 6318-6325.

(25) Flach, C.; Brauner, J.; Mendelsohn, R. Calcium-ion interactions with insoluble phospholipid monolayer films at the a/w interface external reflection-absorption IR studies. Biophys. J. 1993, 65 (5), 1994-2001.

(26) Stefaniu, C.; Brezesinski, G.; Möhwald, H. Polymer-capped magnetite nanoparticles change the $2 \mathrm{D}$ structure of DPPC model membranes. Soft Matter 2012, 8 (30), 7952-7959.

(27) Löbbecke, L.; Cevc, G. Effects of short-chain alcohols on the phase behavior and interdigitation of phosphatidylcholine bilayer membranes. Biochim. Biophys. Acta 1995, 1237, 59-69.

(28) McIntosh, T. J.; Mc Daniel, R. V.; Simon, S. A. Introduction of an interdigitated gel phase in fully hydrated phosphatidylcholine bilayers. Biochim. Biophys. Acta 1983, 731, 109-114.

(29) Vierl, U.; Löbbecke, L.; Nagel, N.; Cevc, G. Solute effects on the colloidal and phase behavior of lipid bilayer membranes: ethanoldipalmitoylphosphatidylcholine mixtures. Biophys. J. 1994, 67, 10671079.

(30) Nuhn, P.; Brezesinski, G.; Dobner, B.; Förster, G.; Gutheil, M.; Dörfler, H.-D. Synthesis, calorimetry, and X-ray diffraction of lecithins containing branched fatty acid chains. Chem. Phys. Lipids 1986, 39, 221-236.

(31) Serrallach, E. N.; Dijkman, R.; de Haas, G. H.; Shipley, G. G. Structure and thermotropic properties of 1,3-dipalmitoyl-glycero-2phosphocholine. J. Mol. Biol. 1983, 170, 155-174.

(32) Tirrell, D. A.; Turek, A. B.; Wilkinson, A. A.; McIntosh, T. J. Observation of an interdigitated gel phase in dipalmitoylphosphatidylglycerol bilayers treated with ionene-6,6. Macromolecules 1985, 18, $1512-1513$. 